\title{
Development of a fast valve for mitigating disruptions in tokamaks
}

\author{
A. Savtchkov, ${ }^{\text {a) }}$ K. H. Finken, and G. Mank \\ Institut für Plasmaphysik, Forschungszentrum Jülich, Association KFA-EURATOM, Member of the Trilateral \\ Euregio Cluster (TEC), 52425 Jülich, Germany
}

(Received 10 May 2002; accepted for publication 3 July 2002)

\begin{abstract}
In support of our disruption mitigation profram, a fast gas valve has been constructed and tested on TEXTOR at FZJ Juelich. Its main features have been shown to be: (1) rapid response time: $0.5 \mathrm{~ms}$; (2) amount of injected gas: variable, 2-1000 mbar $\times 1$; (3) linear dependence of the number of injected particles on the gas pressure; (4) capability of working in a strong magnetic field; (5) sort of gas: any. The valve has the standard CF 35 flange, commonly used in vacuum engineering. All the components that have contact with vacuum were made of stainless steel, except for the closing aluminum piston. To prevent gas leaking directly from the bottles to the experimental vessel there are also two safety valves, closing the bottles before the shot. The required control equipment includes a high power supply and the combined controller for the safety valves and baratrons, both being able to work with TTL control signals. During tests and experiments on TEXTOR and ASDEX-Upgrade, the valve showed successful operation with three gas types: $\mathrm{He}, \mathrm{Ne}, \mathrm{Ar}$. (C) 2002 American Institute of Physics. [DOI: 10.1063/1.1505106]
\end{abstract}

\section{INTRODUCTION}

The most advanced device of fusion machines is the tokamak type. Tokamaks are axisymmetric toroidal systems and therefore relatively simple. For insulating the plasma thermally from the walls, tokamaks require a toroidal magnetic field, which is generated by toroidal magnetic field coils, a poloidal magnetic field, which results from a toroidal current flowing in the highly conducting plasma, and a vertical field, again generated by a set of coils. For engineering reasons, the toroidal magnetic field amounts to a few Tesla in nearly all tokamaks. In order to achieve a stable plasma, the other two field components have to be about one order of magnitude smaller. The plasma current is required both to generate the poloidal confinement field and to heat the plasma (ohmic heating). In small and medium size machines, it amounts to 0.1-1 MA while in the biggest devices reaches up to $7 \mathrm{MA}$. In a future fusion reactor, a plasma current of 15-20 MA will be necessary. Since the plasma current is an abundant source of free energy, an unfavorable current distribution can lead to the creation of internal plasma modes and to enhanced losses of energy and particles or, in the worst case, to an abrupt termination of the plasma current. This process is called disruption.

Disruptions are highly undesired events both for medium size tokamaks but in particular for large ones. In current large size machines, they have already caused significant machine damage. ${ }^{1,2}$ Disruptions must be avoided because they create excessive heating during the energy quench phase and during the current decay phase, generate strong forces on vessel components due to the halo current in the current decay phase, lead to a release impurity from the wall and redeposit it at other areas which may reduce the chance for a successful start of the subsequent discharge, and create ener-

${ }^{a)}$ Electronic mail: a.savtchkov@fz-juelich.de getic runaway electrons with energies increasing with machine size, which are considered to be very dangerous for the next step machines (e.g., ITER). The situation is predicted to be more severe in future devices such as ITER, where the heat loads and forces will be up to two orders of magnitude larger than in present devices.

The typical warning signals for a naturally developing disruption are related to the development of MHD modes when reaching the operational limits. The warning time prior to the disruption can amount to several hundred milliseconds. Proposals have been put forward to start actions during this time that avoid disruptions or destroy the plasma intentionally, e.g., by killer pellets, ${ }^{3}$ liquid jets, ${ }^{4}$ gas puffs, external helical fields, ${ }^{5}$ external negative loop voltage, etc.

In order to mitigate the effects of disruptions, we propose fast gas puffing. Gas puffing has some advantages compared to killer pellets: the gas flow is very fast if the valve is positioned immediately at the vessel and the gas diffuses filling the whole vessel and leaving no space for the development of runaway electrons. For a gas, we prefer helium because of the high recycling and the low sticking probability in the walls; however, other gases or mixtures may prove to be more favorable.

Very crucial for the mitigation is a fast reaction of the valve to a disruption. In favorable cases, one may have a warning time of about $100 \mathrm{~ms}$ given by the development of MHD modes or other signatures. In this case, one can influence on the whole disruption including the energy quench. In less favorable cases, the disruption develops so quickly that only the thermal quench or the voltage spike of the disruption can serve as a trigger. These disruptions can be still mitigated, if the gas is injected very rapidly.

In this contribution, we demonstrate a valve which starts to open $0.5 \mathrm{~ms}$ after a trigger signal and is fully open after 1 $\mathrm{ms}$. The valve is activated by an eddy current induced on an aluminum stem which opens the valve. It is built without 


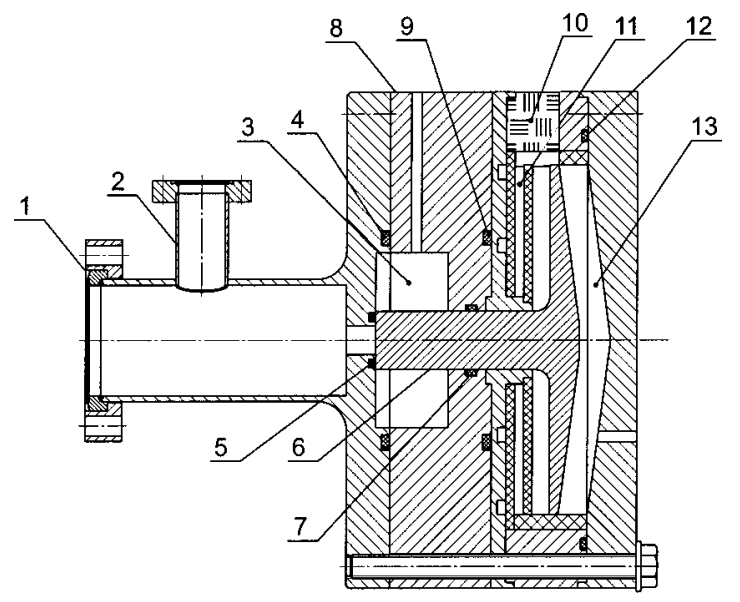

FIG. 1. Cross section drawing of the fast valve. (1) CF 35 flange; (2) pressure measurement flange; (3) working reservoir; (4), (5), (7), (9), (12) o-rings; (6) piston; (8) case; (10) HV coil input (filled with epoxy); (11) coil; (13) closing gas volume.

ferromagnetic materials and can be operated close to the vessel in the environment of the tokamak fields. The amount of injected gas can be varied by the volume of the gas reservoir $\left(5-250 \mathrm{~cm}^{3}\right)$ and the pressures there (typically a few bar). This amount is sufficiently high to induce quickly a density limit disruption but low enough not to damage pumping (e.g., cryopumping). The high pressure in the reservoir guarantees a fast gas stream and a fast reaction time. The valve has been applied successfully on TEXTOR and on ASDEXupgrade.

\section{CONSTRUCTION OF THE VALVE}

Figure 1 shows a cross section of the valve: An aluminum stem (6) seals a fueling gas reservoir (3) from the discharge vessel. The stem is activated to open the valve by the eddy currents induced by a current flowing in a pancake-type coil (11). The electrical current is supplied by a capacitor of $C_{\text {load }}=0.180 \mathrm{mF}$ charged at a voltage of up to $U_{\text {load }}=2 \mathrm{kV}$; the circuit is switched by an ignitron after a trigger signal. The fueling gas reservoir can be varied (by machining) between 5 and $250 \mathrm{ml}$. The fueling pressure should be high enough, enabling a fast gas flow into the discharge; the working pressure is selected between 1 and 30 bar. O-ring (5) seals separate the reservoir from the discharge chamber, and (7) the reservoir from a back volume (13); the pressure in this back volume guarantees a fast closing of the stem after its activation. Towards the discharge chamber, the valve is equipped with a high vacuum CF 35 flange (1) in order to obey the normal tokamak requirements. An additional measurement flange (2) was foreseen as well. The images of the complete valve and of the internal parts are shown in Figs. 2(a) and 2(b), respectively. The total length of the valve amounts to $20 \mathrm{~cm}$ and its diameter to $17 \mathrm{~cm}$.

An important feature of the valve's present design is the avoidance of any ferrite material, enabling it to be installed very close to a tokamak with its full magnetic field. On ASDEX-upgrade, the valve has operated successfully in the full field of $2 \mathrm{~T}$. As pointed out in Sec. I, there is only a limited amount of time between the moment when a disrup-

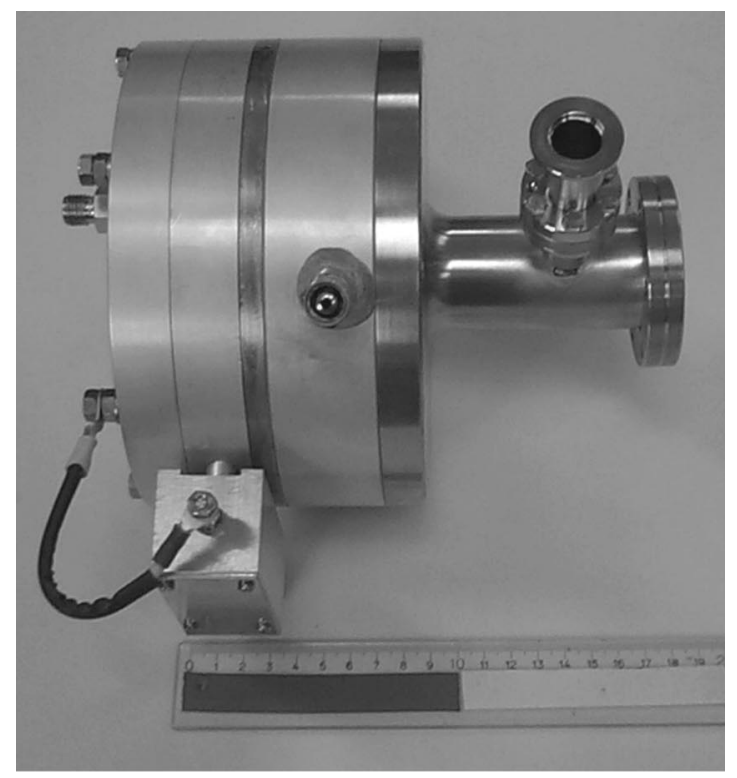

(a)

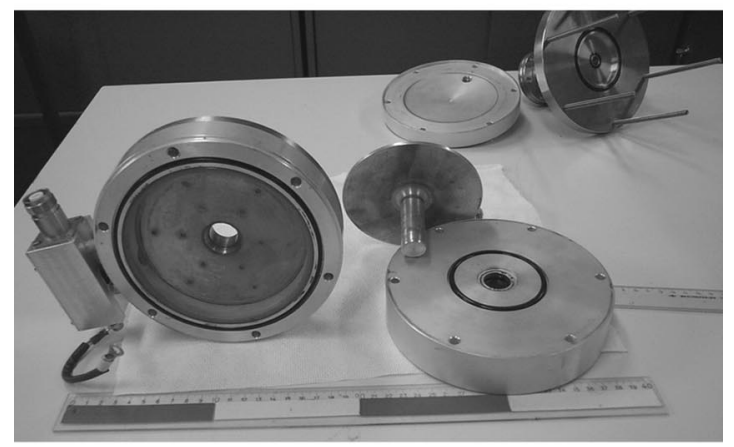

(b)

FIG. 2. Images of the fast valve: (a) general view, (b) disassembled to the component parts.

tion is recognized and the start of the first disruptive phase. Mounting the valve directly on a tokamak vessel results in a faster reaction time since there is no distance separating the valve from the vessel. Other valves use a ferromagnetic stem, which is activated by a coil. A valve with ferromagnetic stem is smaller and has a higher coupling to the electric circuit; but its disadvantage is that the tokamak magnetic field saturates the ferrite stem and it cannot operate directly at the machine.

\section{VALVE OPERATION AND CHARACTERISTICS}

In order to check the valve performance, a test bench has been assembled as indicated in Fig. 3. The test bench includes the fast valve (6) attached to a vacuum volume (11), pressure meters-baratrons (5),(7),(14), a turbopump (16), a high voltage supply (4), and gas bottles (1),(9). Several safety valves (2), (8) are positioned to avoid the situation of a whole gas bottle being released into a discharge volume upon failure of the fast valve. These pneumatically activated valves are located close to the fast valve so that the extra gas reservoir is minimized. One valve (10) is located to pumpout the working volume if the type of gas has to be changed. A controller (3), that unites the functions of supplying the elec- 


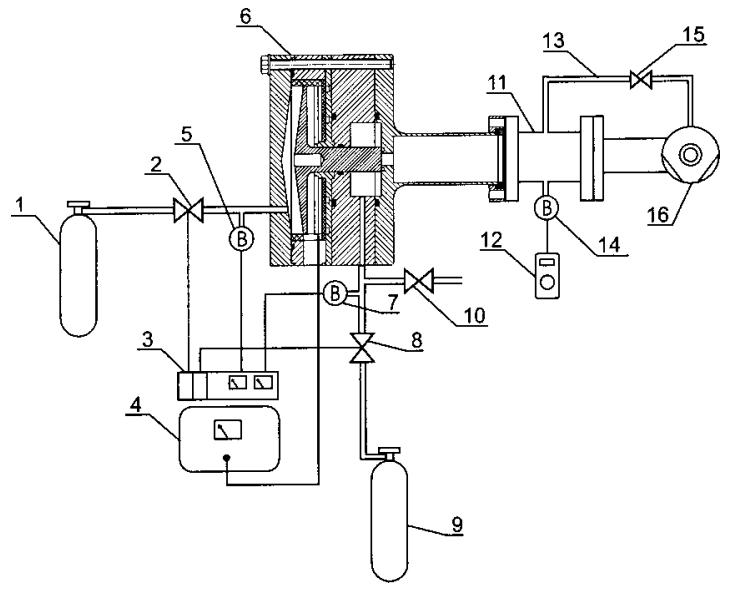

FIG. 3. Test equipment scheme. (1),(9) gas bottles; (2),(8) safety valves; (3) safety valves control, power supply and indication for the baratrons; (4) fast valve high voltage power supply; (5),(7),(14) baratrons; (6) fast valve; (10) pumping valve; (11) target volume; (12) digital voltmeter; (13) bypass; (15) hand valve; (16) turbopump.

tromagnetic valves and baratrons with the power and displaying pressure readouts is assembled in a standard 19-in.Eurocard case. The coil of the fast valve is fed from a capacitor storage (4) using an ignitron high current switch. Both devices are controlled either manually or by a TTL signal. The typical flow diagram during the operation in the laboratory can be reflected in Table I as follows:

First the valve is activated which provides a gas pressure on the stem and closes it off from the discharge vessel (row 1 in the Table I). This step is followed by the refilling of the reservoir by the working gas up to the desired pressure by opening valve 8 (row 2). A high voltage pulse accelerates the stem, which opens the fast valve 6 for about $18 \mathrm{~ms}$ (row 3). After a shot, the injected gas is pumped out from the target volume (11) through the hand valve (15) and by-pass (13).

For the actual experiments on tokamaks, a similar valve switching sequence is performed, except for step 4. Step 1 has to be executed only occasionally. The valve is so tight that the pressure remains for days without refilling. During the tests, the following characteristics were considered: (a) the amount of injected gas, (b) the gas throughput and the reaction time, (c) reliability of operation.

To determine how much gas flows from the working volume for each shot, a scan over the gas pressure has been made. There are two possibilities to calculate the number of injected particles: one can directly measure the pressure increase in the target volume after the shot or derive it know-

TABLE I. Valves states on the different stages of operation.

\begin{tabular}{lccccc}
\hline \hline & \multicolumn{3}{c}{ Fast valve } & & Hand \\
\multicolumn{1}{c}{ Event } & Valve 2 & 6 & Valve 8 & Valve 10 & 15 \\
\hline (1) Closing pressure on & open & closed & closed & closed & closed \\
(2) Working pressure on & closed & closed & open & closed & closed \\
(3) Shot & closed & open & closed & closed & closed \\
(4) Pumping out & closed & closed & closed & closed & open \\
(5) Pumping the & closed & closed & closed & open & closed \\
working volume out & & & & & \\
\hline
\end{tabular}

${ }^{\mathrm{a} O p t i o n a l .}$

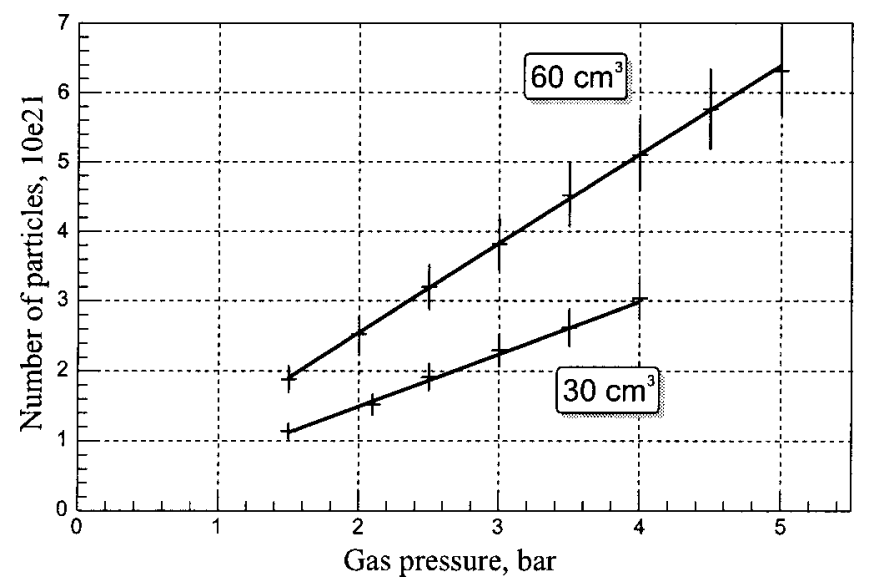

FIG. 4. Number of injected particles vs gas pressure for two different working volumes (note the pressure is in absolute units).

ing the values of the gas pressure before the injection and the working reservoir volume. The calibration curves obtained in the former way for two different cases (namely, for the 30 and $60 \mathrm{~cm}^{3}$ volumes) are shown in Fig. 4. The other parameters are $1.5 \mathrm{kV}$ to activate the valve coil and 5 bar helium for closing. The absolute accuracy of the amount of the injected gas is about $10 \%$, mainly given by the irregular shape of the target volume (11), Fig. 3, into which the gas was released. For the operational range, the amount of the injected gas is proportional to the gas in the filling reservoir. Experiments on ASDEX-upgrade (with the total vessel volume $14 \mathrm{~m}^{3}$ ) have shown that already about 30 mbar 1 (7.5 $\times 10^{20}$ particles) of a gas is sufficient to generate reliably the disruption; on the other hand, the amount of the injected gas is low enough not to shut the pumping system (e.g., turbopumps) down or provoke a detrimental heat load at cryopumps.

The figure of quality of the valve is the gas throughput and the reaction time of the valve. Piezoelectric valves ${ }^{6}$ have an ideal short response time, but their throughput is too small. Therefore a valve with a piston ${ }^{7,8}$ is required that gives sufficient clearance for the gas flow. The most direct response to the action of the valve is the pressure rise measurement in the test chamber after the shot. However, normal pressure gauges are unable to detect the pressure increase in the pressure range involved: ionization gauges or Penning gauges react fast, but they go into saturation at moderate pressures; baratrons have a reaction time of milliseconds and are therefore too slow. In order to obtain the opening characteristic of the valve, we utilize the following method: we insert a glass fiber into the bore hole which leads to the filling reservoir, then we illuminate the glass fiber and detect the light at the valve exit by mounting a photomultiplier (RGA 1P28) at the CF flange of the valve. The light from the glass fiber is reflected many times diffusely at the reservoir walls; therefore the multiplier signal is directly proportional to the opening of the valve. Represented in Figs. 5(a) and 5(b) are the initiation of this opening with extended time scale and the full process, respectively. One sees that the valve starts to open $0.5 \mathrm{~ms}$ after the trigger signal. After about $1 \mathrm{~ms}$, it is nearly completely open and after $18 \mathrm{~ms}$ is 

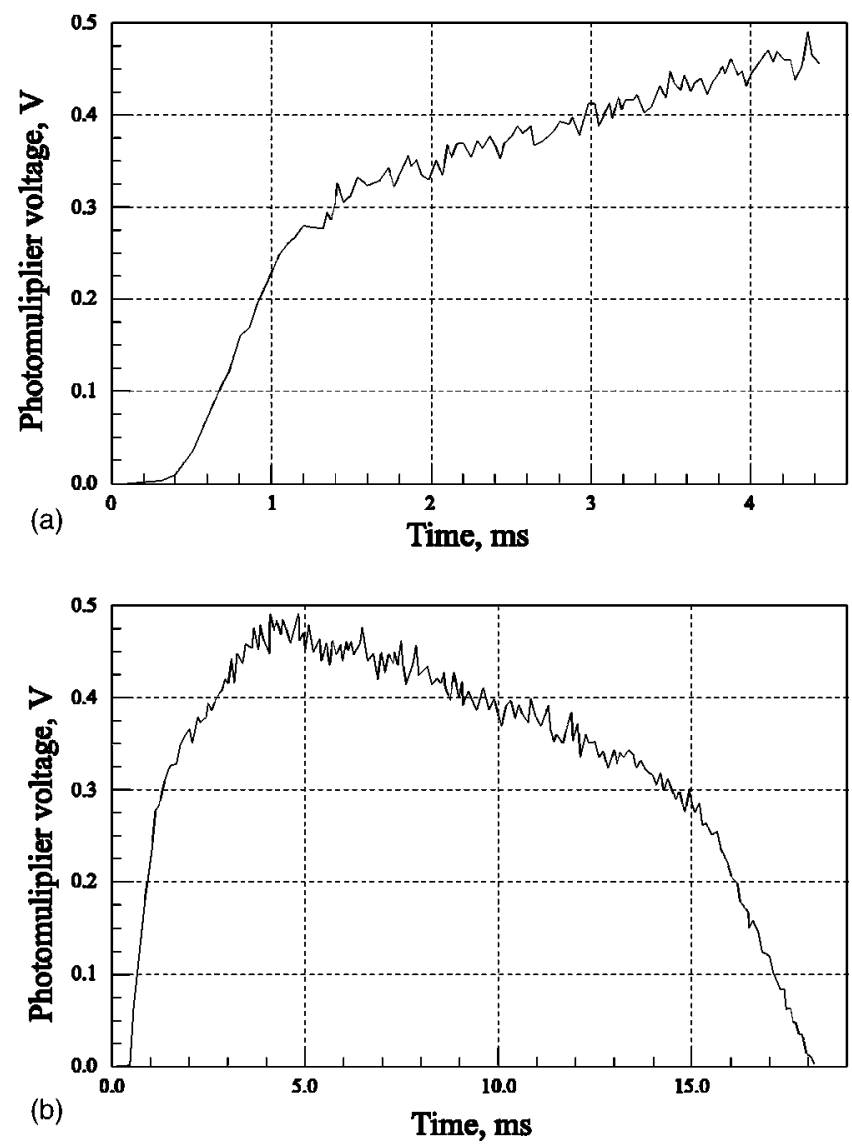

FIG. 5. Opening characteristics of the fast valve. (a) Initial stage with the extended time scale, (b) full process.

closed again due to the repulsive pressure in the back volume.

The curve of the electrical current through the valve coil is of interest as well. Figure 6 gives the plot measured by the standard pulse current instrument AEG ISM-R type 131 connected to the corresponding amplifier AEG type 406. The current maximum is reached after $400 \mu$ s and then the signal decays with the rate of a critically damped oscillation. Since the opening of the valve starts at about the maximum of the valve current, i.e., the stem reaches its maximum kinetic energy also close to this time, the energy transfer from the electrical circuit to mechanical energy is close to its maximum. This means, the capacitance $\mathrm{C}$ is well chosen for the given inductance of the valve. The electrical circuit is switched by an ignitron; this tube switches only one polarity

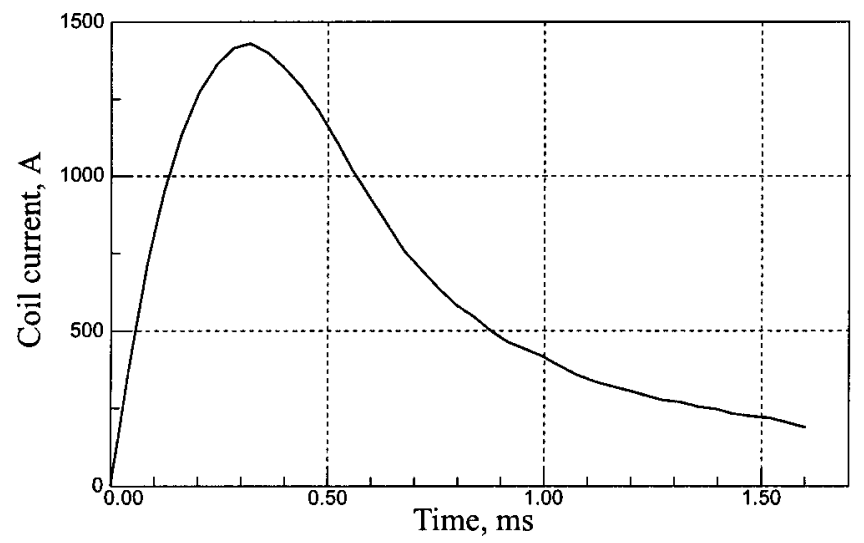

FIG. 6. Trace of the electrical current through the valve coil.

of the current. The fact that the valve current is critically damped is, therefore, very favorable because a less damped circuit would generate high voltages when the current passes through zero. This high induced voltage has proven to be dangerous for the circuit because it may create shorts in the pancake coil and damage it.

To check whether the valve is reliable over a long operation period and whether it remains vacuum tight, continuous cyclic testing has been undertaken. A pressure of $2.5 \mathrm{bar}$ helium was applied for both pushing the closing stem and injecting gas. $2 \mathrm{kV}$ voltage was used for activating. Shots occurred at a frequency of one shot every $40 \mathrm{~s}$, determined by the charging time of the capacitance and the pumping rate of the turbopump. The pressure signal was measured by a baratron in the injected volume. In total, about 1000 shots have been performed within the framework of this test and no change in the character of operation has been found. Up to now, about 500 more gas puffs have been done during experimental programs again without any failure.

${ }^{1}$ G. Federici, C. H. Skinner, J. N. Brooks, J. P. Coad, C. Grisolia, A. A. Haasz, V. Philipps, C. S. Pitcher, J. Roth, W. R. Wampler, and D. G. Whyte, Nucl. Fusion 41, 1967 (2001).

${ }^{2}$ ITER Physics Basis, Nucl. Fusion 39, 2137 (1999).

${ }^{3}$ S. C. Jardin, G. L. Schmidt, E. D. Fredrickson, K. W. Hill, J. Hyun, B. J. Merrill, and R. Sayer, Nucl. Fusion 40, 923 (2000).

${ }^{4}$ M. N. Rosenbluth, S. V. Putvinski, and P. B. Parks, Nucl. Fusion 37, 955 (1997).

${ }^{5}$ R. Yoshino and S. Tokuda, Nucl. Fusion 40, 1293 (2000).

${ }^{6}$ D. Marescaux, Rev. Sci. Instrum. 65, 2412 (1994).

${ }^{7}$ J. Kriesel, R. Prohaska, and A. Fisher, Rev. Sci. Instrum. 62, 2372 (1991).

${ }^{8}$ J. C. Thomas, D. Q. Hwang, R. D. Horton, J. H. Rogers, and R. Raman, Rev. Sci. Instrum. 64, 1410 (1993). 